\title{
Graphene Oxide Nanoribbons: Improved Synthesis and Application in MALDI Mass Spectrometry
}

\author{
Qian Liu, Mengting Cheng, Jing Wang, and Guibin Jiang*[a]
}

\begin{abstract}
Graphene nanoribbon is a novel variety of graphene with high length-to-width ratio and straight edges. Herein, we report an improved method for the synthesis of graphene oxide nanoribbons (GONRs) from longitudinal unraveling of multiwalled carbon nanotubes by means of a one-step, one-pot pressurized oxidation reaction. The obtained GONRs were characterized by different techniques. Furthermore, owing to their unique properties such as strong optical absorption and good water dispersibility, we show that GONRs can be used as an excellent matrix or probe in matrix-assisted or surface-enhanced laser
\end{abstract}

desorption/ionization mass spectrometry (MALDI or SELDI MS) for the first time. In MALDI MS, GONRs generated significantly higher signals than conventional organic matrix and other graphene-based matrices in the detection of lowmass compounds. We also demonstrate the use of GONRs as a sensitive SELDI probe for simultaneous detection of multiple small molecules and profiling of small molecules in complex environmental samples, thus revealing its application potential in rapid screening of low-mass pollutants in complex media.

\section{Introduction}

Matrix-assisted laser desorption/ionization time-of-flight mass spectrometry (MALDI-TOF MS) provides a simple and highthroughput analytical approach for high molecular mass species; however, its application with small molecules is seriously hindered by poor sensitivity and matrix ion interference in low-mass regions. Surface-enhanced laser desorption/ionization time-of-flight mass spectrometry (SELDI-TOF MS) is a variant mode of MALDI-TOF MS. ${ }^{[1]}$ SELDI-TOF MS uses a probe for extraction, preconcentration, desorption, and ionization of the target analytes, thereby avoiding the use of organic matrix and improving the analytical sensitivity. The difference between MALDI and SELDI is the introduction of the probe. An ideal SELDI probe should be able to serve as both a highly efficient extractor and effective MALDI matrix for the target analytes. In recent decades, SELDI-TOF MS has achieved great progress in clinical proteomics, ${ }^{[2,3]}$ but its application in other fields has yet to be explored.

Graphene (G), a one-atom-thick carbon nanosheet with many exceptional properties, has shown great promise for both fundamental and industrial applications. ${ }^{[4-7]}$ Specifically, G has emerged as a good matrix for MALDI-TOF MS owing to its universal and frequency-independent optical absorption prop-

[a] Dr. Q. Liu, M. Cheng, J. Wang, Prof. G. Jiang

State Key Laboratory of Environmental Chemistry and Ecotoxicology

Research Center for Eco-Environmental Sciences

Chinese Academy of Sciences, 18 Shuangqing Road

Haidian District, Beijing 100085 (China)

E-mail:gbjiang@rcees.ac.cn

Supporting information for this article is available on the WWW under http://dx.doi.org/10.1002/chem.201406280. erties and high energy-transfer efficiency. ${ }^{[8-15]}$ However, G often suffers from aggregation, which might greatly compromise its excellent properties that are achieved only with individual sheets and reduce its performance in MALDI-TOF MS. ${ }^{[14]}$ Graphene nanoribbons (GNRs), elongated strips of $G$ with high length-to-width ratio and straight edges, represent a novel variety of $G^{[16-20]}$ GNRs are different from normal $G$ in terms of synthetic method, chemical structure, and geometry. Unlike G, which is normally prepared from graphite, the most facile method to obtain GNRs is longitudinal unzipping of carbon nanotubes (CNTs) by means of chemical oxidation to form graphene oxide nanoribbons (GONRs). ${ }^{[20-22]}$ The GONRs then undergo a chemical reduction process to finally produce GNRs. The unique properties of GNRs can bring about new advantages in applications. For example, GNRs can be used as a versatile material in electronics owing to their width-dependent electronic properties. ${ }^{[18,19]}$ However, synthesis of GNRs or GONRs is still not a straightforward task, and their applications are so far limited.

We noticed that the unique properties of GONRs make them particularly suitable for use as a matrix in MALDI-TOF MS. Because GONRs are longitudinally unzipped from CNTs by means of chemical oxidation, the oxidation sites are supposed to mainly locate at the edges of GONRs without sacrificing the integrity of basal planes (as depicted in Figure 1A). ${ }^{[21,23]}$ Thus, GONRs can preserve the high optical absorption and energytransfer efficiency for MALDI. Meanwhile, the oxygen-containing functionalities in GONRs can prevent their aggregation in solvents and on MALDI target. These properties inspired us to study the application of GONRs in MALDI MS. To the best of our knowledge, the use of GONRs or GNRs in LDI MS is hitherto unreported. 


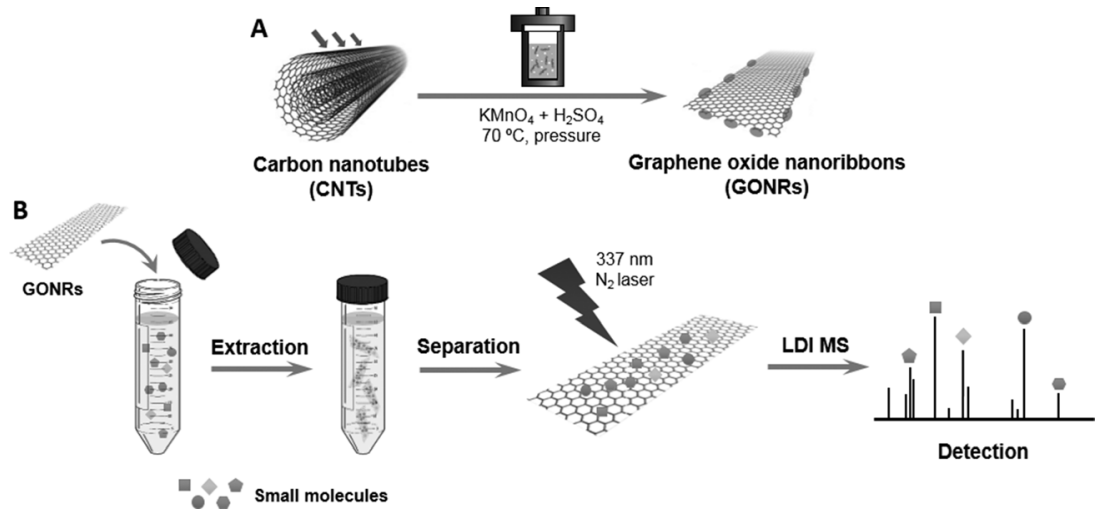

Figure 1. Scheme showing $A$ ) the procedures for the synthesis of GONRs and B) the detection of small molecules by SELDI-TOF MS using GONRs as a probe. In (A) the gray spots indicate the oxidation sites in GONRs.

see Table 1). In addition, this is a one-step, one-pot synthesis, therefore it is more facile and safer than the other methods. We have tested CNTs with different outer diameters as starting materials for GONRs and found that the yield of GONRs increased along with the CNT diameter. This is probably because wide CNTs consist of more stacked layers of graphene sheets than narrow ones, and thus unraveling wide CNTs can produce more GONRs.

The obtained GONRs showed

Herein, we report an improved method for the synthesis of GONRs and show a novel aspect of application for GONRs. The GONRs were synthesized by means of a one-step, one-pot pressurized oxidation of multiwalled carbon nanotubes (MWCNTs; see Figure 1A). We show that GONRs can be used as a valuable matrix for MALDI-TOF MS. We demonstrate the distinct superiority of GONRs over other G-based materials and conventional organic matrix in MALDI-TOF MS. The effects of diameter of the pristine CNTs were investigated. GONRs were also applied as a SELDI probe for simultaneous detection of multiple small molecules and profiling of small molecules in real complex environmental samples (Figure 1B), thus revealing the possibility of using GONR-based SELDI probes for rapid screening of low-mass pollutants.

\section{Results and Discussion}

\section{Synthesis of GONRs by pressurized oxidation of CNTs}

The previously reported methods for the synthesis of GONRs from MWCNTs mainly include the oxidation of MWCNTs by $\mathrm{KMnO}_{4}$ and $\mathrm{H}_{2} \mathrm{SO}_{4}$ at an elevated temperature in an open system. ${ }^{[20-22]}$ These methods require elaborate operations, and the yields are usually not satisfactory. Furthermore, the exothermic process produces a great deal of toxic gases. Here, we transferred the oxidation reaction into a sealed Teflon-lined stainless-steel autoclave (Figure 1A). This simple alteration can eliminate the loss of reactants in the gaseous state (e.g., $\mathrm{KMnO}_{4}$ ) and allows the reaction to proceed under pressurized conditions, thus ensuring high yields of GONRs (96.3-120.7\%;

\begin{tabular}{|c|c|c|c|}
\hline GONR & Pristine CNT & Diameter of CNT [nm] & Yield [\%] \\
\hline GONR-1 & CNT-1 & $10-20$ & 96.3 \\
\hline GONR-2 & CNT-2 & $20-30$ & 97.6 \\
\hline GONR-3 & CNT-3 & $30-50$ & 108.1 \\
\hline GONR-4 & CNT-4 & $>50$ & 120.7 \\
\hline
\end{tabular}

[a] The yield is the mass ratio of the final solid product to the raw CNTs. excellent water dispersibility, and no aggregates were observed in their dispersions $\left(1 \mathrm{mg} \mathrm{mL}^{-1}\right)$ after storage for ten months at room temperature. GNRs could be obtained by the reduction of the as-prepared GONRs with hydrazine as described previously. ${ }^{[2]}$ However, if GONRs were reduced to GNRs, their water dispersibility would be decreased significantly, which was unfavorable for their use as a MALDI matrix or SELDI probe.

\section{Characterization of GONRs}

The as-prepared GONRs were characterized by different techniques. On the basis of TEM measurements (Figure 2), the degree of consecutive tube opening in the CNTs increases along with the diameter of CNTs. This trend is consistent with the yield of GONRs in Table 1. For CNT-4 (o.d. $>50 \mathrm{~nm}$ ), the CNTs can be completely unraveled to form GONRs by means of pressurized oxidation. The width of the GONRs increases to more than $120 \mathrm{~nm}$. The full-scan and C1s XPS spectra of the
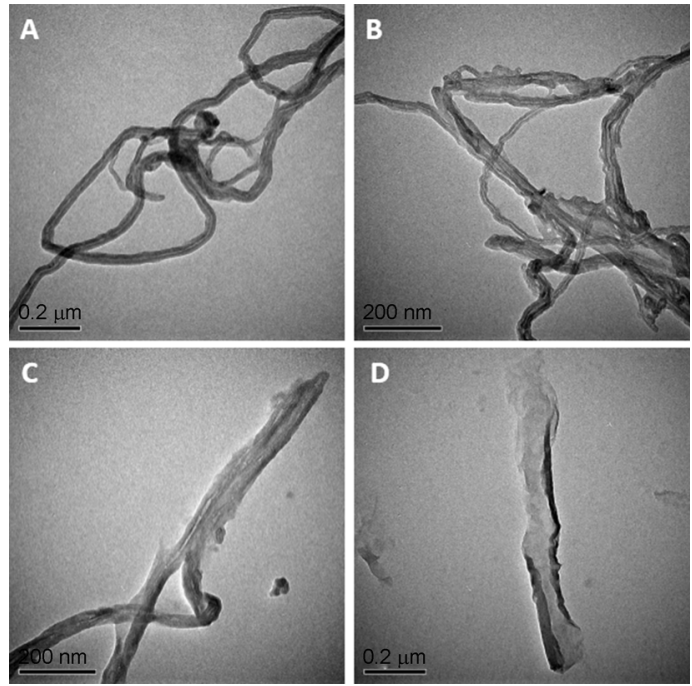

Figure 2. TEM images of GONRs unzipped from CNTs of different diameters. A) Pristine (NT-4, B) GONR-1, C) GONR-3, and D) GONR-4. The diameters of pristine CNTs are given in Table 1. 
GONRs and pristine CNTs are compared in Figures S1 and S2 of the Supporting Information. The results also indicate that the degree of oxidation in GONRs increases with the diameter of the pristine CNTs. In the following text, GONR/CNT refers to GONR-4/CNT-4 if not otherwise noted.

Figure $3 \mathrm{~A}$ compares the optical absorption spectra of GONRs, G, and graphene oxide (GO). The $\mathrm{G}$ used here was obtained from chemical exfoliation of graphite followed by hydrazine reduction, also called chemically converted graphene
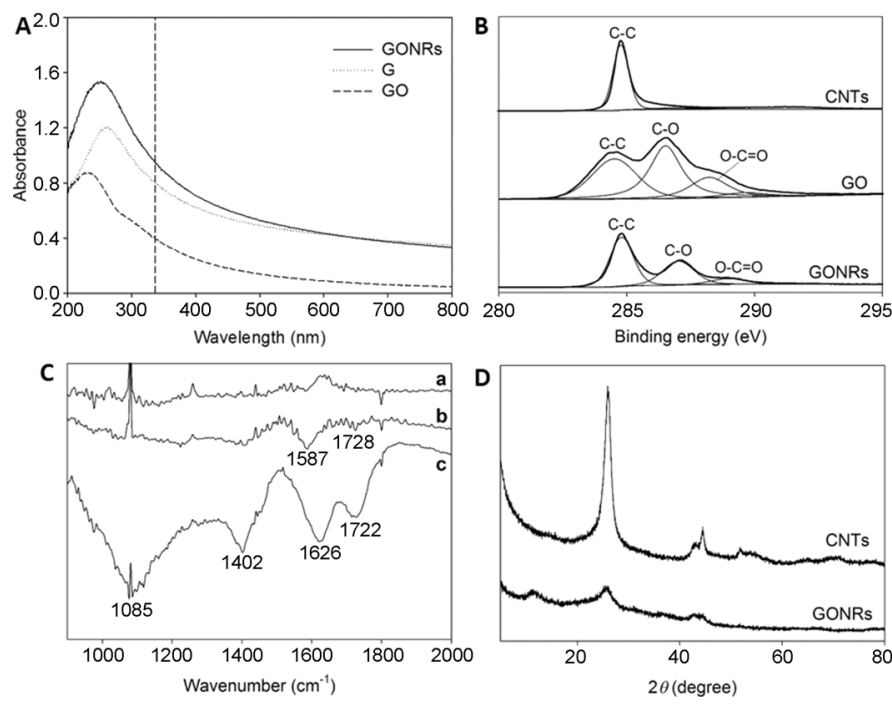

Figure 3. Characterization of GONRs. A) UV-visible absorption spectra of GONRs, G, and GO. B) C1s XPS spectra of CNTs, GO, and GONRs. C) FTIR spectra of a) CNTs, b) GONRs, and c) GO. D) XRD patterns of GONRs and CNTs.

(CCG). The absorbance of GONRs is significantly higher than that of GO. Notably, the absorbance of GONRs is even higher than that of $G$ at $337 \mathrm{~nm}$ (i.e., the laser wavelength used in MALDI), thus suggesting a higher energy absorption capability of GONRs in MALDI. The optical absorption properties actually reflect the integrity of the $\pi$-conjugated network in $G$ sheets. ${ }^{[24]}$ Thus, this result is consistent with the hypothesis that the majority of oxidation sites locate at the edges of GONRs and that the graphite structures in GONRs can be mainly preserved during the longitudinal unzipping of CNTs. ${ }^{[23]}$

In the C1s XPS spectra (Figure 3B), GONRs yield three main components at $284.8,287.0$, and $289.0 \mathrm{eV}$ assigned to $\mathrm{C}-\mathrm{C}, \mathrm{C}-$ $\mathrm{O}$, and $\mathrm{O}-\mathrm{C}=\mathrm{O}$ bonds, respectively. ${ }^{[20]}$ The peaks of oxygen functionalities of GONRs are much lower than those in GO, thus indicating a lower degree of oxidation in GONRs than in GO. This was also verified by FTIR. In the FTIR spectrum of GO (Figure 3C), the peak at $1722 \mathrm{~cm}^{-1}$ is assigned to the $\mathrm{C}=\mathrm{O}$ stretching of carboxyl groups, and the absorption at $1626 \mathrm{~cm}^{-1}$ is due to the $\mathrm{O}-\mathrm{H}$ bending vibration, epoxide groups, and skeletal ring vibrations. ${ }^{[14,25]}$ These peaks are also observed for GONRs but much weaker than those observed for GO. The structures of GONRs and pristine CNTs were also characterized by XRD (Figure 3D and Figure S3 in the Supporting Information). CNTs show a predominant peak at $26.0^{\circ}$, which corresponds to a $d$ spacing of $3.4 \AA$. The graphite (002) spacing increases with the degree of oxidation. After oxidation to GONRs, the peak at $26.0^{\circ}$ is greatly decreased, and a new peak appears at $12.0^{\circ}$, which corresponds to a $d$ spacing of $7.4 \AA$. The broadening of the peak indicates the disruption of the well-ordered stacking structure in MWCNTs by oxidation. Overall, the characterization results indicate that GONRs have been successfully oxidized but the degree of oxidation is relatively low, which means few defects in the basal planes.

\section{GONRs as a matrix in MALDI-TOF MS}

We evaluated the performance of GONRs as a matrix in MALDI-TOF MS with four types of environmental pollutantsorganochlorine pesticide pentachlorophenol (PCP), endocrine disrupter estradiol (E2), brominated flame retardant 2,2',4,4'tetrabromodiphenyl ether (BDE-47), and tetrabromobisphenol A (TBBPA)—as model analytes (molecular weight 266-544; see Figure 54 in the Supporting Information for chemical structures).

Figure 4 shows the comparison of GONR with pristine CNT, $\mathrm{G}, \mathrm{GO}$, and conventional organic matrix $\alpha$-cyano-4-hydroxycinnamic acid (CHCA). Generally, for all four analytes, GONR gives the highest MS signals among the tested matrices. The baselines for GONR are quite clean with very little noise peaks, thus indicating that GONRs are sufficiently stable to resist fragmentation in MALDI. For PCP (Figure 4A)-except for CHCA, which gives no signals-other matrices produce strong peaks of $[\mathrm{M}-\mathrm{H}]^{-},[\mathrm{M}-\mathrm{H}-\mathrm{Cl}]^{-}$, and $[\mathrm{M}-\mathrm{H}-\mathrm{Cl}-\mathrm{CO}]^{-}$at $\mathrm{m} / \mathrm{z} 264.1,229.1$, and 201.1. For E2 (Figure 4B), the peak of $[M-H]^{-}$is detected at $\mathrm{m} / \mathrm{z} 270.5$ for GONR, CNT, G, and CHCA, but only noise peaks are observed for GO. Notably, the peak intensity for GONR is much higher than that for other matrices. For BDE-47 (Figure $4 \mathrm{C}$ ), the triple peaks of $\left[\mathrm{M}-\mathrm{C}_{6} \mathrm{H}_{3} \mathrm{Br}_{2}\right]^{-}$at $\mathrm{m} / \mathrm{z} 250.1$ are detected for all the matrices except CHCA. The intensity for GONR is 3.0 times higher than for CNT and 2.2 times higher than for $\mathrm{G}$. The results for TBBPA are similar to that of BDE-47 (Figure 4D). The multiple peaks of $[M-H]^{-},[\mathrm{M}-\mathrm{OH}]^{-}$, $[\mathrm{M}-\mathrm{H}-\mathrm{Br}]^{-},[\mathrm{M}-\mathrm{OH}-\mathrm{Br}]^{-}$, and $[\mathrm{M}-2 \mathrm{OH}-\mathrm{Br}]^{-}$are detected at $\mathrm{m} / \mathrm{z} 542.6,526.4,462.4,446.5$, and 419.0 , respectively. The peak intensity of $[\mathrm{M}-\mathrm{H}]^{-}$for GONR is $36.4,8.7$, and 8.6 times higher than that for CNT, G, and GO, respectively. The limits of detection (LODs; signal-to-noise ratio $(\mathrm{S} / \mathrm{N})=3$ ) of the analytes on GONR and $G$ matrix are compared in Table 2. These results clearly demonstrate that GONR is a much better matrix than the other tested materials. The superiority of GONR is particularly prominent in the detection of less-polar compounds.

We then investigated the morphology of GONR film formed on the MALDI target by means of SEM (Figure S5 in the Supporting Information). The homogeneity of the matrix is an important factor that affects the ionization/desorption efficiency in MALDI. ${ }^{[14,26]}$ Conventional organic matrices such as CHCA usually result in "hot spots" owing to a inhomogeneous cocrystallization process with analytes. Among the tested materials, GO and GONRs could form uniform and flat films, but in the CNT film many entanglements were observed. The G film showed the poorest homogeneity with the $G$ sheets aggregating or restacking to discrete graphite flakes during 

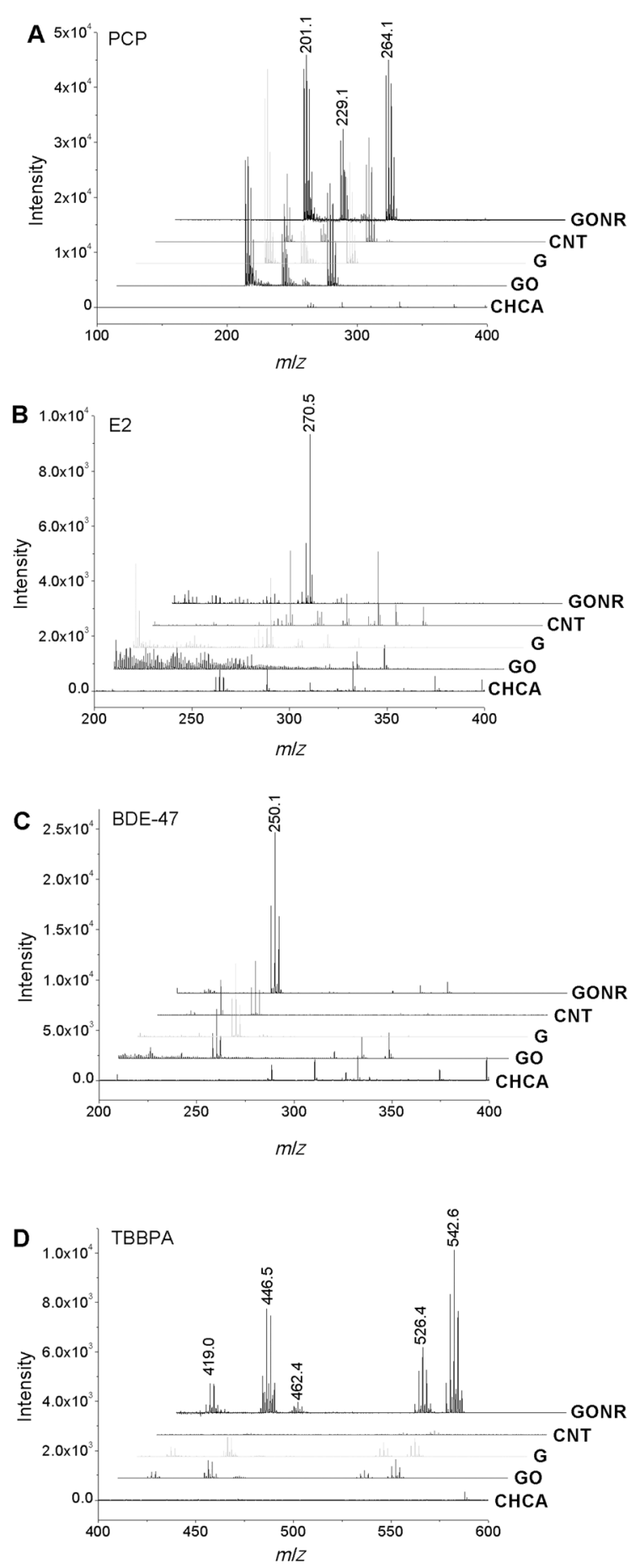

Figure 4. Comparison of performance of different matrices (GONR, CNT, G, GO, and $(\mathrm{HCA})$ in MALDI-TOF MS detection of A) P(P, B) E2, C) BDE-47, and D) TBBPA. Analyte concentration: $0.1 \mathrm{mg} \mathrm{mL}^{-1}$.

the drying process. These results indicate that aggregation of GONRs is suppressed in the matrix owing to their good water dispersibility.

From the discussion above, we can conclude that it is the unique synthetic method and structures of GONRs that bring about their high performance in MALDI-TOF MS. The longitudinal unzipping of CNTs can satisfactorily maintain the $\pi$-conjugated structure in GONRs, thereby resulting in strong laser
Table 2. $L O D s(S / N=3)^{[a]}$ and RSDs $(n=20)$ for detection of PCP, E2, BDE47 , and TBBPA by MALDI-TOF MS using GONRs and $G$ as matrix.

\begin{tabular}{lcccc} 
Analyte & \multicolumn{2}{c}{ GONR } & \multicolumn{2}{c}{ G } \\
& LOD [pg] & RSD [\%] & LOD [pg] & RSD [\%] \\
\hline PCP & 0.8 & 1.7 & 0.5 & 10.1 \\
E2 & $1.7 \times 10^{2}$ & 22.3 & $3.3 \times 10^{2}$ & 32.1 \\
BDE-47 & 40 & 6.4 & 50 & 21.3 \\
TBBPA & $3.9 \times 10^{2}$ & 7.7 & $2.5 \times 10^{3}$ & 38.7
\end{tabular}

[a] The LODs were obtained based on the highest feature peaks of the analytes.

energy absorption; at the same time, the oxygen-containing functionalities in GONRs can help their dispersibility in solutions and on MALDI target, thus retaining the large surface area of GONRs and allowing high energy-transfer efficiency. These properties are similar to those of nitric acid oxidized graphene, ${ }^{[14]}$ but GONRs are easier and more cost-effective to obtain. Furthermore, GONRs are more uniform in geometry than normal $G$ sheets, which is favorable for obtaining reproducible analytical results. As listed in Table 2, the shot-to-shot RSDs $(n=20)$ at different locations on GONR matrix range from 1.7 to $22.3 \%$, whereas these values on the $\mathrm{G}$ matrix are in the range of $10.1-38.7 \%$.

The effects of the diameter of pristine CNTs on the performance of the GONR matrix were also studied. As shown in Figure 5, in the detection of TBBPA, the MS signal increases

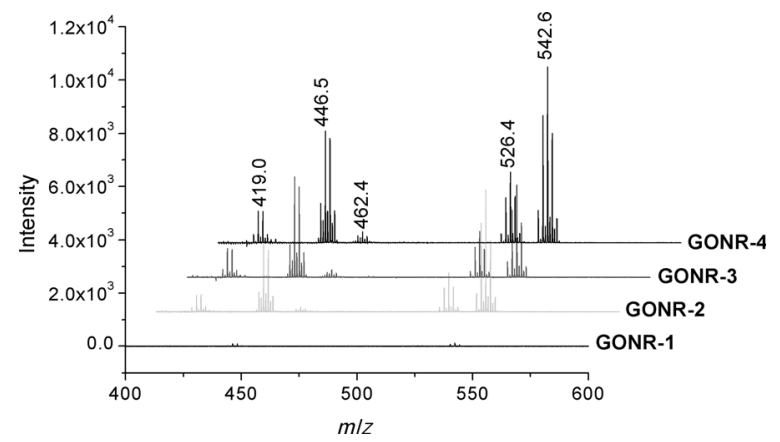

Figure 5. Comparison of performance of GONRs unraveled from MWCNTs with different diameters in MALDI-TOF MS detection of TBBPA. Analyte concentration: $0.1 \mathrm{mg} \mathrm{mL}^{-1}$.

along with the diameter of the pristine CNT. Similar results were also obtained for the other analytes (Figure S6 in the Supporting Information). As demonstrated above, the degree of tube opening in CNTs and the yield of GONRs increase with the expansion of the CNT diameter. Since GONRs were more efficient than CNTs in MALDI MS, better performance was obtained with the GONRs unraveled from wider CNTs. Thus, GONR-4 was used in the following SELDI-TOF MS experiments.

\section{GONRs as a SELDI probe for small molecules}

In addition to a MALDI matrix, we also attempted to use GONRs as a SELDI probe (Figure 1B). GONRs can offer distinct 
advantages as a SELDI probe. In addition to high performance in MALDI as demonstrated above, GONRs can also serve as an excellent extractor for the target analytes. GONRs originate from CNTs and have a similar chemical structure and morphology to $\mathrm{G}$, thus keeping the excellent extraction capability of $\mathrm{G}$ and CNTs toward a wide spectrum of compounds. ${ }^{[27-30]}$

We tested the use of GONR-4 as a probe for detection of multiple small molecules in aqueous samples. As shown in Figure 6, three types of small molecules (BDE-47, E2, and TBBPA) can be simultaneously detected by using the GONR
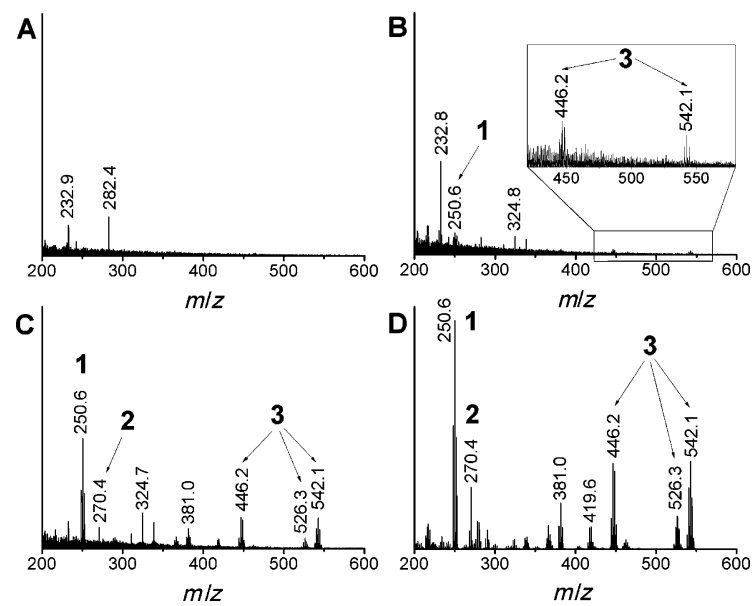

Figure 6. Simultaneous detection of 1) BDE-47, 2) E2, and 3) TBBPA in spiked water samples by SELDI-TOF MS using GONRs as a probe. Spike concentration: A) 0, B) 10, C) 100 , and D) $500 \mathrm{ng} \mathrm{mL}^{-1}$.

probe at concentrations lower than $10 \mu \mathrm{g} \mathrm{mL}^{-1}$. The MS signals positively correlate with the analyte concentration. Compared with the signal intensities obtained by direct MALDI-TOF MS analysis, the SELDI-TOF MS produces significantly higher signals for less-polar analytes, thus indicating that the GONR probe has a specific enrichment for these compounds. For instance, the LODs for BDE-47 and TBBPA on SELDI-TOF MS were estimated to be 3.3 and $4.4 \mathrm{ng} \mathrm{mL}^{-1}$ (equivalent to 17 and $22 \mathrm{pg}$ ), respectively. The ability to simultaneously detect different types of compounds is of great value for the analysis of complex samples.

High-throughput analysis of small molecules in complex samples is currently a great challenge. To demonstrate the possibility of using this method for rapid screening of low-mass pollutants, we applied it in the profiling of small molecules in real environmental samples. The influent and effluent samples from a wastewater treatment plant (WWTP) spiked with BDE-47 were analyzed by SELDI-TOF MS. As shown in Figure 7, a set of peaks were detected from $\mathrm{m} / \mathrm{z} 100$ to 300 . We did not identify each peak; however, the spiked BDE-47 can be easily detected at $\mathrm{m} / \mathrm{z} 250.6 / 250.3$, thus indicating that the results are reliable. Notably, on the basis of the profiling, it can be recognized that the number and intensities of peaks in the effluent samples are reduced relative to those in the influent samples, thus indicating that a part of small molecules were removed by the wastewater treatment process. It should be noted that the profiling is based on the extraction and desorption/ionization on GONRs, and thereby it might not
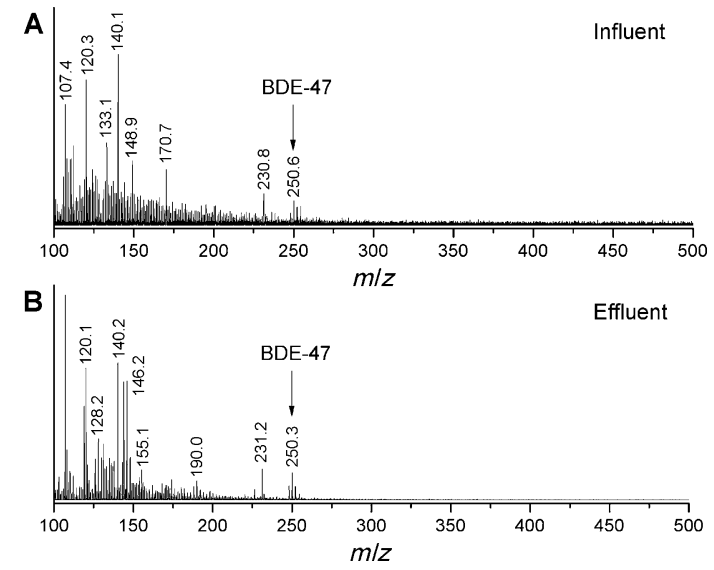

Figure 7. Profiling of small molecules in A) influent and B) effluent samples from a WWTP by SELDI-TOF MS using GONRs as probe. The samples were spiked with BDE-47 at $10 \mathrm{ng} \mathrm{mL}^{-1}$.

be an exhaustive profiling of small molecules. Considering that GONRs can interact with molecules through different mechanisms such as hydrophobic interactions, $\pi-\pi$ interactions, electrostatic interactions, or hydrogen bonding, a considerable number of small molecules, especially less polar and aromatic compounds, can be detected. Accordingly, the GONR-based SELDI probe provides a simple and usable tool for rapid screening of low-mass pollutants in complex media.

\section{Conclusion}

In summary, we have shown that GONRs can be used as an excellent MALDI matrix or SELDI probe in the analysis of small molecules owing to their unique properties. GONRs can be facilely prepared using a one-step, one-pot pressurized oxidation method from the unraveling of MWCNTs with high yields. The as-prepared GONRs show good water dispersibility and strong optical absorption properties. In MALDI-TOF MS, GONRs show much better performance than a conventional organic matrix, CNTs, and other G-based matrices. The GONRs unraveled from wide CNTs work better than those from narrow CNTs. Furthermore, GONRs can be used as a highly efficient SELDI probe for simultaneous detection of multiple small molecules and profiling of small molecules in complex media. Therefore, it might provide a promising tool for high-throughput analysis of small molecules. With such simplicity and high efficiency, we believe that GONR-based LDI techniques retain the possibility of playing a role in future "omics" research such as exposomics and metabolomics. ${ }^{[31-33]}$

\section{Experimental Section}

\section{Synthesis of GONRs}

GONRs were longitudinally unzipped from MWCNTs by using a pressurized oxidation method (Figure 1A). Briefly, MWCNTs (75 mg) were placed in a Teflon-lined stainless-steel autoclave that contained $\mathrm{KMnO}_{4}(375 \mathrm{mg})$ and $\mathrm{H}_{2} \mathrm{SO}_{4}(75 \mathrm{~mL})$. The mixture was heated at $70^{\circ} \mathrm{C}$ for $3 \mathrm{~h}$. Afterward, cooled water $(200 \mathrm{~mL})$ and $\mathrm{H}_{2} \mathrm{O}_{2}$ 
( $5 \mathrm{~mL}, 30 \%$ ) were added to the mixture. The solids were washed thoroughly with $1 \mathrm{~m} \mathrm{HCl}$ solution and water, and then dispersed in water followed by sonication for 20 min. Finally, the products were collected and air-dried.

\section{Characterization of materials}

TEM images were captured using a Hitachi H-7500 transmission electron microscope (Tokyo, Japan). SEM images were captured using a Hitachi S-3000N scanning electron microscope. XPS spectra were obtained using an AXIS Ultra DLD X-ray photoelectron spectrometer (Kratos, Manchester, UK) with $\mathrm{Al}_{\mathrm{K} \alpha} \mathrm{X}$-ray radiation as the X-ray source excitation. FTIR spectra were obtained using a JASCO Fourier transform infrared spectrometer (Victoria, B.C., Canada). The samples were mixed and ground with $\mathrm{KBr}$, then pressed into transparent disks for measurement. UV-visible absorption spectra were obtained using a Shimadzu UV-3600 UV/Vis-NIR spectrophotometer (Kyoto, Japan). XRD patterns were obtained using a PANalytical X'Pert PRO X-ray diffraction system (Almelo, Netherlands).

\section{MALDI- and SELDI-TOF MS}

For the direct MALDI-TOF MS analysis, the matrix $(5 \mu \mathrm{L})$ was placed on the MALDI target and was blended with the analyte solution. For the GONR-based SELDI-TOF MS analysis, the process is shown in Figure 1B. The GONR probe was dispersed in water at $1 \mathrm{mg} \mathrm{mL}^{-1}$. For extraction, the probe dispersion $(50 \mu \mathrm{L})$ was added into the sample solution $(1 \mathrm{~mL})$, and the mixture was shaken at a rate of $300 \mathrm{rmin}^{-1}$ for $30 \mathrm{~min}$. The probe was then concentrated by centrifugation at $\times 15000 \mathrm{~g}$ for $30 \mathrm{~min}$. The supernatant was discarded, and the precipitate $(5 \mu \mathrm{L})$ was deposited on a stainlesssteel MTP target frame III (Bruker Daltonics) for detection. Negative-ion MALDI-TOF MS was performed using a Bruker Daltonics Autoflex III Smartbean MALDI-TOF mass spectrometer in reflector mode controlled by FlexControl software. A $337 \mathrm{~nm}$ nitrogen laser with a frequency of $100 \mathrm{~Hz}$ was used. The FlexAnalysis 3.0 software was used for data processing.

\section{Acknowledgements}

This work was co-supported by the Strategic Priority Research Program of the Chinese Academy of Sciences (XDB14010400), the National Natural Science Foundation of China (21321004, 21107120, 21377141, and 21422509) and an Excellent Young Scientist Grant of the Chinese Academy of Sciences.

\section{Keywords: carbon - graphene - mass spectrometry} nanostructures $\cdot$ oxidation

[1] N. Tang, P. Tornatore, S. R. Weinberger, Mass Spectrom. Rev. 2004, 23, 34-44.

[2] C. Liu, J. Biomed. Biotechnol. 2011, 2011, 245821.
[3] F. Simpkins, J. A. Czechowicz, L. Liotta, E. C. Kohn, Pharmacogenomics 2005, 6, 647-653.

[4] K. S. Novoselov, V. I. Fal'ko, L. Colombo, P. R. Gellert, M. G. Schwab, K Kim, Nature 2012, 490, 192-200.

[5] D. Bitounis, H. Ali-Boucetta, B. H. Hong, D. H. Min, K. Kostarelos, Adv. Mater. 2013, 25, 2258-2268.

[6] L. P. Biró, P. Nemes-Incze, P. Lambin, Nanoscale 2012, 4, 1824-1839.

[7] S. J. Guo, S. J. Dong, Chem. Soc. Rev. 2011, 40, 2644-2672.

[8] X. L. Dong, J. S. Cheng, J. H. Li, Y. S. Wang, Anal. Chem. 2010, 82, 6208 6214.

[9] Y. Liu, J. Y. Liu, C. H. Deng, X. M. Zhang, Rapid Commun. Mass Spectrom. 2011, 25, 3223-3234.

[10] B. Gulbakan, E. Yasun, M. I. Shukoor, Z. Zhu, M. X. You, X. H. Tan, H. Sanchez, D. H. Powell, H. J. Dai, W. H. Tan, J. Am. Chem. Soc. 2010, 132, $17408-17410$.

[11] C. Shi, J. Meng, C. Deng, Chem. Commun. 2012, 48, 2418-2420.

[12] M. H. Lu, Y. Q. Lai, G. N. Chen, Z. W. Cai, Anal. Chem. 2011, 83, 3161 3169.

[13] J. Y. Liu, Y. Liu, M. X. Gao, X. M. Zhang, J. Am. Soc. Mass Spectrom. 2012, 23, 1424-1427.

[14] Q. Liu, M. T. Cheng, G. B. Jiang, Chem. Eur. J. 2013, 19, 5561-5565.

[15] L. A. L. Tang, J. Z. Wang, K. P. Loh, J. Am. Chem. Soc. 2010, 132, 1097610977.

[16] D. K. James, J. M. Tour, Acc. Chem. Res. 2013, 46, 2307-2318.

[17] M. Terrones, A. R. Botello-Méndez, J. Campos-Delgado, F. López-Urías, Y. I. Vega-Cantú, F. J. Rodríguez-Macías, A. L. Elías, E. Munoz-Sandoval, A. G. Cano-Márquez, J.-C. Charlier, H. Terrones, Nano Today 2010, 5 , $351-372$.

[18] S. Dutta, S. K. Pati, J. Mater. Chem. 2010, 20, 8207-8223.

[19] L. Jiao, L. Zhang, X. Wang, G. Diankov, H. Da, Nature 2009, 458, 877 880.

[20] D. V. Kosynkin, A. L. Higginbotham, A. Sinitskii, J. R. Lomeda, A. Dimiev, B. K. Price, J. M. Tour, Nature 2009, 458, 872-875.

[21] A. L. Higginbotham, D. V. Kosynkin, A. Sinitskii, Z. Sun, J. M. Tour, ACS Nano 2010, 4, 2059-2069.

[22] D. K. James, J. M. Tour, Macromol. Chem. Phys. 2012, 213, 1033-1050.

[23] C. S. Xiang, P. J. Cox, A. Kukovecz, B. Genorio, D. P. Hashim, Z. Yan, Z. W. Peng, C. C. Hwang, G. D. Ruan, E. L. G. Samuel, P. M. Sudeep, Z. Konya, R. Vajtai, P. M. Ajayan, J. M. Tour, ACS Nano 2013, 7, 10380-10386.

[24] D. Li, M. B. Muller, S. Gilje, R. B. Kaner, G. G. Wallace, Nat. Nanotechnol. 2008, 3, 101-105.

[25] C. Y. Hou, Q. H. Zhang, M. F. Zhu, Y. G. Li, H. Z. Wang, Carbon 2011, 49, $47-53$.

[26] X. Z. Zhou, Y. Y. Wei, Q. Y. He, F. Boey, Q. C. Zhang, H. Zhang, Chem. Commun. 2010, 46, 6974-6976.

[27] Q. Liu, J. B. Shi, G. B. Jiang, TrAC Trends Anal. Chem. 2012, 37, 1-11.

[28] Q. Liu, J. Shi, J. Sun, T. Wang, L. Zeng, G. Jiang, Angew. Chem. Int. Ed. 2011, 50, 5913-5917; Angew. Chem. 2011, 123, 6035-6039.

[29] Q. Liu, J. B. Shi, L. X. Zeng, T. Wang, Y. Q. Cai, G. B. Jiang, J. Chromatogr. A 2011, 1218, 197-204.

[30] L. M. Ravelo-Pérez, A. V. Herrera-Herrera, J. Hernández-Borges, M. Á. Rodríguez-Delgado, J. Chromatogr. A 2010, 1217, 2618-2641.

[31] P. J. Lioy, Environ. Health Perspect. 2010, 118, 1081-1090.

[32] C. P. Wild, A. Scalbert, Z. Herceg, Environ. Mol. Mutagen. 2013, 54, 480 499.

[33] T. J. Athersuch, Bioanalysis 2012, 4, 2207-2212.

Received: November 28, 2015

Published online on February 16, 2015 